\title{
LOS MECANISMOS DE NEGOCIACIÓN INTERNACIONAL PARA IMPULSAR LA PRODUCTIVIDAD ECUATORIANA
}

\author{
INTERNATIONAL NEGOTIATION MECHANISMS TO BOOST \\ ECUADORIAN PRODUCTIVITY
}

\author{
Derli Francisco Álava Rosado', Pedro Alexander Avilés Almeida ${ }^{2}$, Walter Iván Navas Bayona ${ }^{3}$ \\ 1 - Universidad Laica Eloy Alfaro de Manabí, Manta, Ecuador \\ 2- Universidad Estatal de Guayaquil, Guayaquil, Ecuador \\ 3 - Universidad San Gregorio de Portoviejo, Ecuador
}

1. Email: derli.alava@uleam.edu.ec ORCID: https://orcid.org/0000-0001-9906-5784

1. Email: pedro.avilesal@ug.edu.ec ORCID: https://orcid.org/0000-0001-5207-2645

1. Email: winavas@sangregorio.edu.ec ORCID: https://orcid.org/0000-0002-5646-1821

Recibido: 10/05/2021 Aceptado: 22/07/2021

Para Citar: Álava Rosado, D. F., Avilés Almeida, P. A., \& Navas Bayona, W. I. (2021). Los mecanismos de negociación internacional para impulsar la productividad ecuatoriana. Revista Publicando, 8(31), 26-35. https://doi.org/10.51528/rp.vol8.id2231

\begin{abstract}
Resumen:
La desaceleración del crecimiento económico del Ecuador debido a la caída del precio del barril de petróleo evidencia la necesidad de impulsar la productividad económica mediante la promoción de una mayor iniciativa privada sustentada en una política internacional y diversos mecanismos de negociación. Para ello, se realizó una investigación descriptiva bajo el enfoque cuantitativo con una revisión documental de las implicaciones de las negociaciones internacionales en la economía, los indicadores de productividad en el Ecuador y la importancia de la política económica en la productividad. Tras el análisis de estos factores, se encontró que para mejorar la productividad se debe capacitar, asistir técnica y tecnológicamente y brindar acceso a mercados y negocios internacionales a las empresas, apoyar el emprendimiento con políticas económicas que promuevan la innovación y tecnología para incentivar la inversión y relaciones internacionales comerciales.
\end{abstract}

Palabras clave: negociación internacional, productividad, política económica internacional.

\begin{abstract}
:
The deceleration of economic growth of Ecuador due to fall of oil barrel price proof the need to promote economic productivity by promoting a higher private initiative based on an international policy and various negotiating mechanisms. For this, a descriptive investigation was carried out under the quantitative approach with a documentary review of the implications of international negotiations in the economy, indicators of productivity in Ecuador and the importance of economic policy on productivity. After the analysis of these factors, it was found that to improve productivity it must be trained, technically and technologically and provide access to international markets and business to companies, support entrepreneurship with economic policies that promote innovation and technology to encourage investment and international commercial relations.
\end{abstract}

Keywords: international negotiation, productivity, international economic policy. 


\section{INTRODUCCIÓN}

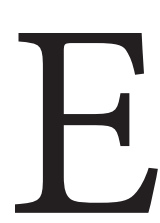

1 2020, la emergencia sanitaria causada por la COVID-19 provocó una recesión que redundó en un repunte de la pobreza, desequilibrios macroeconómicos y evidenció la elevada informalidad, la falta de amortiguadores macroeconómicos, un sistema de salud poco preparado y la falta de acceso a servicios públicos. Esta problemática conlleva a promover la recuperación de la actividad económica y proteger a los grupos más vulnerables reequilibrando las finanzas públicas para evitar el aumento de la deuda pública mientras se mejora sistemáticamente el ambiente de inversiones para favorecer el crecimiento y la creación de empleo de calidad (Banco Mundial, 2020).

El bajo crecimiento económico de Ecuador se ha debido al lento crecimiento de la productividad tanto en el área tecnológica, laboral, industrial, agropecuaria dado que la estructura económica se concentra en el sector de servicios con una cifra de $58.3 \%$, mientras el primario con una participación del $21.4 \%$ y el secundario una cifra de $25.3 \%$ y su dependencia de la exportación, principalmente de petróleo, cuya volatilidad de precios demostró la fragilidad del modelo económico ecuatoriano (Luna, 2014).

Con el objeto de superar tales desequilibrios económicos, Ecuador requiere transitar hacia un modelo de crecimiento con mayor iniciativa privada mediante el fortalecimiento de las finanzas públicas, el impulso de la productividad y el desarrollo del sector privado y la profundización de los avances sociales (Banco Interamericano de Desarrollo, 2020), considerando además otros sectores mayormente productivos como: elaboración de productos de tabaco, fabricación de papel, fabricación de coque y de productos de la refinación del petróleo, fabricación de productos de caucho y plástico, fabricación de otros productos minerales no metálicos, fabricación de metales comunes, fabricación de equipo eléctrico, fabricación de vehículos automotores, remolques y semirremolques.

El crecimiento de una mayor iniciativa privada se sustenta en una política internacional donde se aborda el estudio de problemáticas como las relaciones monetarias, los flujos comerciales y la inversión extranjera, dando cuenta de la interrelación entre la política nacional e internacional cuya interrelación puede explicarse mediante la teoría de los juegos de doble nivel (Nemiña y Zelicovich, 2016).

Las negociaciones internacionales pueden darse no sólo entre los estados sino también entre líderes empresariales como individuos singularmente $\mathrm{o}$ en representación de las organizaciones de naturaleza privada (Calle, 2007). En cualquiera de los casos se está en la obligación de descifrar la cultura de la contraparte con la finalidad de analizar el origen, la naturaleza, las características y orientación de las estrategias en función de la dinámica y el objeto de la negociación.

La inversión por iniciativa de empresas privadas internacionales promueve la producción, entendida como la cantidad total de bienes y servicios que se generan en una economía durante un período determinado de tiempo, combina dos factores disponibles como insumos: capital físico (maquinaria y equipo) y capital humano (fuerza laboral) (Quintana, Mendoza, y Correa, 2014). Si 
aumenta la cantidad de insumos disponibles, la producción se incrementa. Sin embargo, también puede incrementarse cuando los factores de producción se aprovechan de forma más eficiente.

En ese sentido, la productividad consiste en la relación entre la producción obtenida y los recursos utilizados para obtenerla, tratándose de un indicador de eficiencia que relaciona la cantidad de producción con la cantidad de insumos utilizados Carrillo (2013), es decir, la productividad se refiere a la eficiencia con la que una economía emplea sus recursos para generar riqueza. Por lo tanto, la productividad se logra al mantener la misma producción empleando la menor cantidad de recursos o aumentando el nivel de producción con la misma cantidad de recursos.

La productividad se clasifica en productividad física y productividad económica. La productividad física se relaciona con las unidades físicas y la productividad económica con las unidades monetarias de los bienes y servicios producidos y el número de insumos utilizados (Quintana, Mendoza y Correa, 2014). Ambas presentan desventajas en su medición, cuando una economía genera múltiples bienes y servicios de todo tipo se dificulta la medición de la productividad física de unidades distintas de producto y en el caso de la productividad económica se tiende a generar un problema de sobrevaloración, cuando sobre el cálculo de ésta intervienen factores del valor agregado de un producto, como nivel de producción, nivel de insumos, precio del producto y precio de los insumos, obteniendo un aumento aparente de la productividad (Carrillo, 2013). En consecuencia, la productividad económica no es el indicador apropiado de eficiencia, pero permite sumar unidades distintas de producto, por lo cual es más fácil de obtener y comparar que la productividad física.

El crecimiento económico causado por un incremento de la productividad, a diferencia del crecimiento causado por un incremento en los insumos, modifica la relación entre producción e insumo, resaltando la eficiencia con la que la economía combina el capital físico y el capital humano para generar valor agregado, cuya medida más usada es el Producto Interno Bruto (PIB), definido como el valor de todos los bienes y servicios finales producidos en un país durante un período determinado de tiempo (Mankiw, 2002).

Por otro lado, cuando el crecimiento económico no puede ser explicado por aumentos en la cantidad de insumos utilizados en el proceso de producción, sino que es el resultado de un incremento en la eficiencia con la que se utilizan o se combinan los factores de producción, se refiere la productividad total de los factores (PTF) (Freire, 2001).

Para distinguir entre el crecimiento atribuible a un incremento en la cantidad de factores empleados y aquella que se debe a mejoras en la productividad, se recurre al modelo tradicional de crecimiento económico de Solow. El modelo tradicional de crecimiento económico de Solow distribuye el crecimiento de la producción entre crecimiento del capital físico, capital humano y productividad total de los factores, dando como resultado que las fuentes del crecimiento del producto son la contribución del capital físico, del capital humano y la productividad total de los factores de forma residual debido al crecimiento económico no atribuido a la acumulación de 
factores, es decir, el remanente de crecimiento que no puede ser explicado por aumentos en la cantidad de capital físico y humano (Carrillo, 2013). En este modelo, los rendimientos constantes significan que un cambio proporcional en la producción se da ante un cambio proporcional en los insumos o, en su defecto, a la productividad total de los factores, produciendo el denominado progreso técnico o tecnológico determinante del crecimiento de la productividad.

La productividad es fundamental en el crecimiento económico de un país, debido a que cuando aumenta la productividad, aumenta la producción por el incremento de la inversión y del consumo privado provocando una reducción de los costos, lo que deriva en el aumento de la oferta real monetaria, reduciendo el tipo de interés y aumentando la demanda de dinero, la inversión y por tanto la demanda de bienes y servicios que incrementa el nivel de empleo y aumento de los salarios (Carrillo, 2013). Cuanto más productiva es una economía, más altos son los ingresos de los trabajadores y más baja la tasa de inflación en el largo plazo. Por consiguiente, la productividad es la base de un crecimiento económico próspero, acompañado de un aumento tanto de la ocupación (empleo) como del ingreso (salario) en términos reales.

El panorama económico se distingue entonces por unas pocas empresas muy productivas $y$ muchas de productividad extremadamente baja. Lamentablemente, los altos índices de informalidad protegen más a las microempresas en Ecuador, que contratan menos de 100 empleados, que a las empresas con más de 100 empleados y con alta capacidad productiva desde el punto de vista de la productividad laboral (Romero, Flores,
Campoverde y Coronel, 2017), sin embargo estas microempresas incrementan la productividad agregada y provocan un cambio estructural importante debido a que ofrecen nuevos productos y servicios que "permiten un dinamismo colateral hacia industrias proveedoras de insumos, esto estimula la competitividad de los mercados y la innovación en todas sus formas dando paso al crecimiento de la economía" (Zamora, 2018, 3).

No obstante, Ecuador ha implementado varias políticas públicas desde el 2011 a partir del Código Orgánico de la Producción, Comercio e Inversiones, por medio del cual se decretó que el Estado es el encargado de "promover el desarrollo productivo mediante la determinación de políticas e incentivos que fomenten la transformación de la matriz productiva, lo cual estimula la competitividad de las empresas y a su vez la producción sostenible" Camino, Armijos y Cornejo (2018), de tal forma que las normativas, instituciones y programas que incentivan la productividad son competencia de las políticas públicas del Ecuador.

En ese sentido, en esta investigación se plantea que el mejoramiento de los mecanismos de negociación internacional impulsa la productividad como clave fundamental para el crecimiento económico del Ecuador.

\section{METODOLOGÍA}

En el presente estudio se pretende analizar los mecanismos de negociación internacional que impulsan la productividad ecuatoriana, para lo cual se realiza una investigación descriptiva bajo el enfoque cuantitativo con una revisión documental de las implicaciones de las negociaciones 
internacionales en la economía, los indicadores de productividad en el Ecuador y la importancia de la política pública en la productividad para finalmente presentar los resultados y conclusiones.

\section{RESULTADOS Y DISCUSIÓN}

Los mecanismos de negociación internacional que pueden emplearse para impulsar la productividad económica de Ecuador pueden tratarse de procesos de internacionalización, que implican la participación y negociación en foros multilaterales y bilaterales, como forma de inserción en el plano internacional; relaciones internacionales, para formalizar un acuerdo, convenio, carta intención, protocolo y contrato, entre otros; gestión y negociación intercultural, para aumentar la frecuencia de las negociaciones entre personas de diferentes países y culturas; relaciones diplomáticas entre el gobierno y cada negociador; como estrategia, para la selección de tácticas y estrategias apropiadas para ser adoptadas durante la negociación; como proceso donde se considera el escenario de la negociación, los negociadores, los asuntos a resolver $y$ las decisiones que los negociadores toman; entornos internacionales, caracterizados por el ambiente de la negociación, las diferencias culturales y subculturales, las diferencias ideológicas, la burocracia, las leyes y los gobiernos extranjeros, la inseguridad financiera, la inestabilidad política y los repentinos cambios económicos y la cultura nacional que influye en los propósitos de las negociaciones internacionales (Calle, 2007). De allí, que estos mecanismos de negociación sean considerados como un medio entre dos o más partes para resolver conflictos, alcanzar consensos o negociar por intereses individuales o colectivos.
Al respecto, el modelo harvardiano de negociación, de autoría de Fisher y Ury, sobre el conjunto de discursos acerca de la resolución negociada consiste en convencer a los entes en litigio de que la búsqueda de un acuerdo implica menos costos que mantener una postura luchadora (Acselrad, das Neves y Muñoz, 2010). En el ámbito de negocios internacionales se llega a modalidades de resolución directa si las partes no cumplen con los convenios establecidos, en cuyo caso se amerita un proceso de conciliación, facilitación, mediación y arbitraje.

De acuerdo con lo anterior, es importante resaltar la complejidad que puede caracterizar la negociación internacional cuando tiende a vincularse a la resolución de conflictos, aunque generalmente se trata de conducir a la realización de acuerdos en el orden comercial (usualmente regulados en contratos), "se trata de un proceso en el cual las partes deciden poner fin a su controversia a procurar un determinado acuerdo sin la mediación de los organismos judiciales y por supuesto del empleo de la violencia para el logro de un determinado fin" (Calle, 2007, p. 31), resumido en la Figura 1.

La política pública de apoyo al emprendimiento por parte del gobierno ecuatoriano se ha enfocado en la creación de normativas, instituciones y programas para facilitar la inclusión económica y el desarrollo de actividades productivas en el sector de la economía popular y solidaria y sectores estratégicos (recursos hídricos, hidrocarburos, minería, electricidad y telecomunicaciones) Zamora (2018), considerando su ubicación (la región costera es más productiva que la región sierra, amazonía e insular, debido a su acercamiento a puertos 
internacionales como el de Guayaquil, Manta y Esmeraldas). Sin embargo, poco es el apoyo de la formación de los empresarios hacia objetivos más claros de productividad, innovación y tecnología.

Por ello es importante que el Estado, sin descuidar los sectores sociales, mantenga constante o incremente las inversiones en los sectores estratégico productivos a través de un mayor gasto público, "atrayendo la inversión extranjera directa y mejorando el clima de cooperación bilateral y multilateral para el desarrollo agrícola y rural, especialmente no reembolsable" Pino, Aguilar, Apolo y Sisalema (2018), con el propósito de reducir la pobreza.

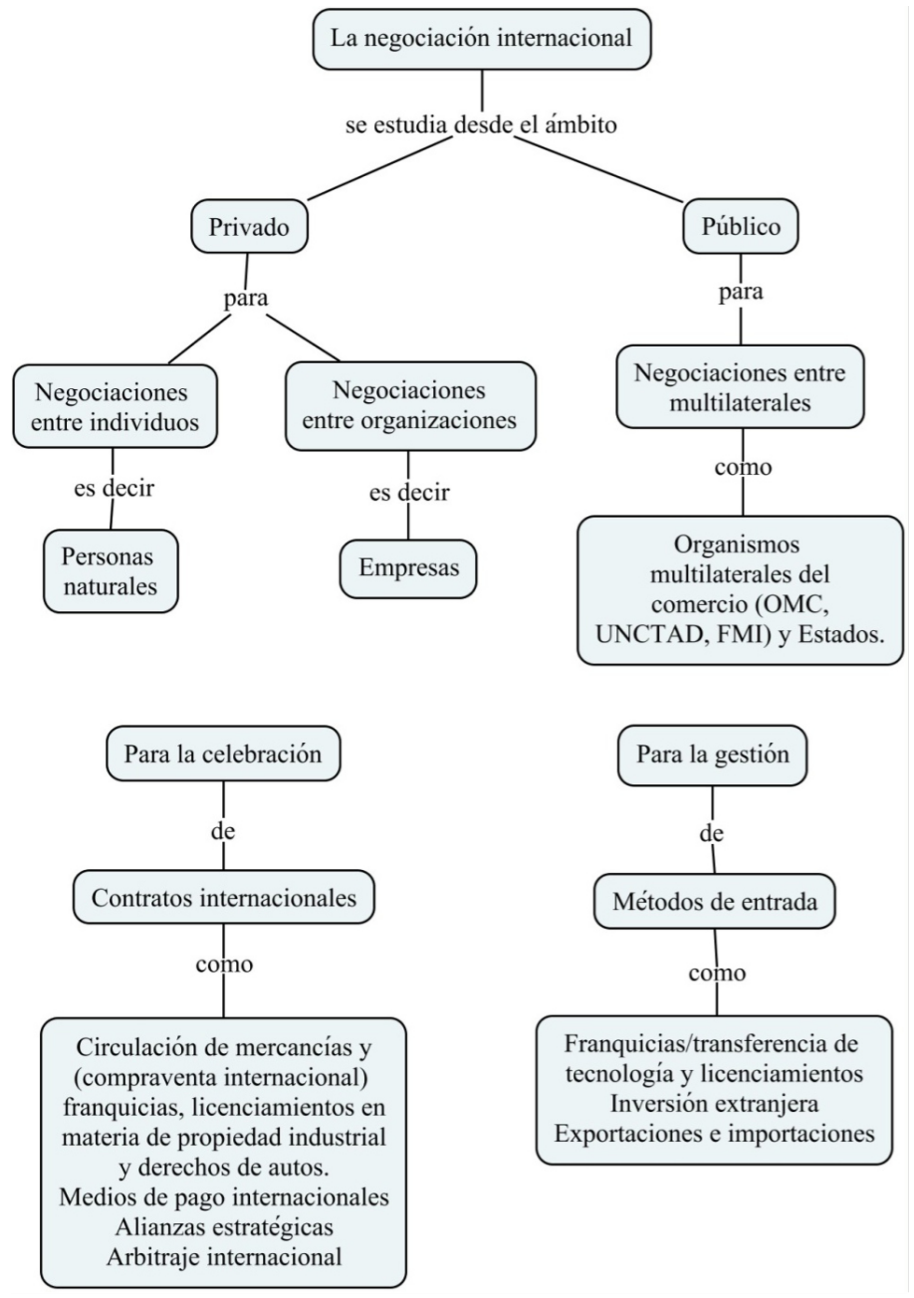

Fuente: Calle (2007).. 
Por otro lado, la productividad total de los factores está estrechamente relacionada con crecimientos o decrecimientos de acuerdo con el PIB, por lo cual se deduce que el aumento de la productividad total de los factores estimula el incremento de otras variables como la inversión, el empleo, el stock de capital concluyendo en un aumento de la producción Camino, Armijos y Cornejo (2018); por ejemplo, las reformas tributarias, la mejora en la eficiencia recaudatoria, la recompra de los bonos Global y el acceso al financiamiento extranjero, "permitiendo la expansión del gasto público que financió inversiones productivas y sociales que mejoraron las condiciones de vida de la población y la competitividad sistémica de la economía" Guzmán (2016) en e Ecuador desde 1990 y 2014.

\section{CONCLUSIONES}

El bajo crecimiento de Ecuador se debe principalmente al reducido incremento de la productividad no sólo a nivel agregado sino a nivel sectorial y laboral, el cual se desempeña mayormente en sectores de baja productividad relativa como el agropecuario y el de los servicios, por lo que se deben estudiar las causas de la baja productividad sectorial y capacitar, asistir técnica y tecnológicamente y brindar acceso a mercados y financiamiento a las empresas para que aumenten su productividad.

La promoción del emprendimiento también es indispensable en el aumento de la productividad ecuatoriana al atraer inversionistas a sectores estratégicos de sus economías con la finalidad de reducir el desempleo, dinamizar la estructura productiva e incrementar el nivel de producción. Estos emprendimientos tienen poco acceso a financiación nacional e internacional y otras limitaciones de tipo político y social, pero estas microempresas deben tener un potencial de inversión al proponer productos innovadores y tecnológicos que correspondan con las políticas nacionales de productividad.

En cuanto a los mecanismos de negociación que deben emplearse para garantizar la mayor productividad, que según los datos más recientes del Banco Mundial (2020), presenta una desaceleración en su crecimiento llegando a ser negativo $(-1,6 \%)$ en 2019 , debido a la caída del precio del barril de petróleo; se consideran necesarios para el desarrollo de negociaciones internacionales privadas entre personas naturales y empresas para la celebración de contratos internacionales como circulación de mercancías y franquicias, licenciamientos en materia de propiedad industrial y derechos de autor, medios de pago internacionales, alianzas estratégicas y arbitraje internacional y para la gestión de métodos de entrada como franquicias, transferencia de tecnología y licenciamientos, inversión extranjera, exportaciones e importaciones.

El esquema de doble nivel puede ser utilizado satisfactoriamente en el estudio de negociaciones internacionales no limitadas exclusivamente a las bilaterales entre estados sino entre organismos internacionales, como el FMI, estados y negociaciones multilaterales; no obstante su aplicación requiere una serie de modificaciones a las políticas económicas de Ecuador.

Las negociaciones internacionales para impulsar la productividad en Ecuador no tratan de limitar el ingreso de productos y servicios al país, 
por el contrario, estos permiten desarrollar otras alternativas por parte de los productores para competir en el mercado nacional e internacional, sino que se pretende que el enfoque de las nuevas negociaciones sirvan para desarrollar mejores productos y servicios que logren posicionar la marca ecuatoriana con un enfoque de calidad, incrementar la inversión y del consumo privado, la demanda de bienes y servicios, el nivel de empleo $\mathrm{y}$ aumento de los salarios.

De igual manera, los mecanismos de negociación internacional implementados para impulsar la productividad ecuatoriana deben ser secuenciales y dinámicos, lo que requiere una planificación estratégica y una ejecución sistemática de acciones que garanticen el cumplimiento de los acuerdos logrados, centrando las negociaciones en transacciones que logren acuerdos basados en el futuro que se pretende perseguir en la economía ecuatoriana, lo cual es competencia de las políticas públicas de Ecuador.

En ese sentido, las políticas públicas basadas en las negociaciones internacionales deberían prestarle mayor atención a las empresas que consumen materias primas internacionales tanto para promover las exportaciones como para generar beneficios empresariales a las importaciones de ciertos insumos de producción que no los produzca Ecuador, o si los produce intensificar la mejora de la calidad y ubicación de dichos insumos en las empresas que producen bienes finales con valor agregado. 


\section{REFERENCIAS BIBLIOGRÁFICAS}

Acselrad, H., das Neves, G.y Muñoz, E. (2010). Inserción económica internacional $\mathrm{y}$ "resolución negociada" de conflictos ambientales en América Latina. EURE (Santiago), 36(107), 27-47.

Banco Interamericano de Desarrollo. (2020). Perspectiva general del Ecuador. Recuperado el 01 de Junio de 2021, de http://iadb.org/paises/ecuador/ perspectiva-general

Banco Mundial. (2020). World Development Indicators (WDI). Recuperado el 01 de Junio de 2021, de http:// datos.bancomundial.org/indicador

Calle, M. (2007). Estado del arte en el estudio de la negociación internacional. Medellín, Colombia: Cuaderno de Investigación de la Universidad EAFIT.

Camino, S., Armijos, G. y Cornejo, G. (2018). Productividad Total de los Factores en el sector manufacturero ecuatoriano: evidencia a nivel de empresas. Cuadernos de economía. 41, 241-261.

Carrillo, D. (2013). Situación de la productividad en América Latina y Ecuador. Trabajo especial de grado de la Universidad.

Freire, M. B. (2001). La productividad total de los factores en el Ecuador: efectos microeconómicos sobre las tasas de ganancia, los precios relativos y los salarios reales y determinantes macroeconómicos de su evolución. Ecuador: Banco Central del Ecuador.
Guzmán, M. (2016). Crecimiento, productividad y cambio estructural en ecuador, 1990-2014. En H. Burchardt, R. Domínguez, C. Larrea y S. Peters, Nada dura para siempre (pp. 159-198). Ediciones Abya-Yala: Quito, Ecuador.

Luna, G. (2014). Ecuador: economía y política de la revolución ciudadana, evaluación preliminar. Apuntes del CENES, 109-134.

Mankiw, N. (2002). Principios de Economía. México: Cengage Learning.

Nemiña, P.y Zelicovich, J. (2016). El análisis de las negociaciones internacionales. Reflexiones metodológicas sobre la aplicación del esquema de doble nivel. Revista POSTData: Revista de Reflexión y Análisis Político, 21(2), 423-452.

Pino, S., Aguilar, H., Apolo, G.y Sisalema, L. (2018). Aporte del sector agropecuario a la economía del Ecuador. Análisis crítico de su evolución en el período de dolarización. Años 2000-2016. Revista ESPACIOS, 1-24.

Quintana, L., Mendoza, M.y Correa, R. (2014). Regiones y Economía en Ecuador. Investigaciones Regionales, (30), 227-228.

Romero, C., Flores, G., Campoverde, J.y Coronel, K. (2017). Microempresas panificadoras en el Azuay (Ecuador) y su productividad. RETOS. Revista de Ciencias de la Administración y Economía, 7(14), 167-188. 
$R$ E V I S T A

Publ i c a n d o

I S S N $13390-9304$

Zamora, C. (2018). La importancia del emprendimiento en la economía: el caso de Ecuador. Espacios, 15. 